\title{
WEAK TOPOLOGIES AND EQUICONTINUITY
}

\author{
DONALD F. REYNOLDS AND JOHN W. SCHLEUSNER ${ }^{1}$
}

\begin{abstract}
Corresponding to each family $F$ of real-valued functions on a set $X$, there is a weakest topology on $X$ for which $F$ is equicontinuous. This equiweak topology is pseudometrizable and provides a characterization of metrizable topologies in terms of point-separating families of real-valued functions.
\end{abstract}

Introduction. Let $X$ be a set and let $F$ be a collection of real-valued functions defined on $X$. In this paper, we show there always exists a weakest topology on $X$ for which $F$ is an equicontinuous family. We define this to be the equiweak topology induced by $F$ and show that such topologies play a role in metrization theory analogous to the role of weak topologies in the theory of completely regular Hausdorff spaces. In particular, we show that a topology is metrizable if and only if it is an equiweak topology induced by a point-separating family of real-valued functions.

We first establish some notation and terminology. All functions are defined on $X$ and are real-valued. The collection of all such functions is denoted by $R^{X}$. The weak topology induced on $X$ by $F \subset R^{X}$ is denoted by $\mathcal{T}_{F}$. The collection $F$ separates points in $X$ if for distinct points $x_{1}, x_{2} \in X$, there is some $f \in F$ such that $f\left(x_{1}\right) \neq f\left(x_{2}\right)$. If $X$ is equipped with a topology, $C(X)$ will denote its collection of continuous functions. A collection $F \subset R^{X}$ is equicontinuous at $x_{0} \in X$ if, given $\varepsilon>0$, there is a neighborhood $U$ of $x_{0}$ so that $\left|f(x)-f\left(x_{0}\right)\right|<\varepsilon$ for all $x \in U$ and all $f \in F$. If $F$ is equicontinuous at $x_{0}$ for each $x_{0} \in X$, then $F$ is simply said to be equicontinuous.

The equiweak topology.

Lemma 1. Let $F \subset R^{X}$ be equicontinuous at $x_{0} \in X$. Then for each $p \in X$, the function $x \rightarrow \sup _{F}\{|f(x)-f(p)| \wedge 1\}$ is continuous at $x_{0}$.

Proof. Fix $p \in X$. Then for any $x \in X$, we have that

$$
\begin{aligned}
& \left|\sup _{F}\{|f(x)-f(p)| \wedge 1\}-\sup _{F}\left\{\left|f\left(x_{0}\right)-f(p)\right| \wedge 1\right\}\right| \\
& \quad \leqslant \sup _{F}\left\{\left|[|f(x)-f(p)| \wedge 1]-\left[\left|f\left(x_{0}\right)-f(p)\right| \wedge 1\right]\right|\right\} \\
& \quad \leqslant \sup _{F}\left\{|| f(x)-f(p)|-| f\left(x_{0}\right)-f(p)||\right\}<\sup _{F}\left\{\left|f(x)-f\left(x_{0}\right)\right|\right\} .
\end{aligned}
$$

Received by the editors November 17, 1978 and, in revised form, November 12, 1979.

AMS (MOS) subject classifications (1970). Primary 54C30, 54E35.

Key words and phrases. Equicontinuity, weak topologies, metrization.

${ }^{1}$ The results in this paper were presented by the first author on March 20, 1980 at the UAB Topology Conference sponsored by the Department of Mathematics at the University of Alabama in Birmingham and the National Science Foundation (Grant \# MCS-7922039). 
But by the equicontinuity of $F$ at $x_{0}$, this last term can be made arbitrarily small in a suitable neighborhood of $x_{0}$.

THEOREM 2. Let $F \subset R^{X}$. Then the following are equivalent:

(i) $F$ is equicontinuous.

(ii) For each $p \in X$, the function $a_{p}$ defined by $a_{p}(x)=\sup _{F}\{|f(x)-f(p)| \wedge 1\}$ is continuous on $X$.

Proof. (i) $\Rightarrow$ (ii). Fix $p \in X$. Let $x_{0} \in X$ and let $\varepsilon>0$ be given. Since $F$ is equicontinuous at $x_{0}$, by Lemma 1 there is a neighborhood $U$ of $x_{0}$ so that $\left|a_{p}(x)-a_{p}\left(x_{0}\right)\right|<\varepsilon$ for all $x \in U$. Thus $a_{p}$ is continuous on $X$.

(ii) $\Rightarrow$ (i). Let $p \in X$ and let $\varepsilon>0$ be given. By the continuity of $a_{p}$ at $p$, there is a neighborhood $U$ of $p$ so that $\left|a_{p}(x)-a_{p}(p)\right|<\varepsilon$ for all $x \in U$. But since $a_{p}(p)=0$, we have that $0 \leqslant a_{p}(x)<\varepsilon$, or equivalently, that

$$
\sup _{F}\{|f(x)-f(p)| \wedge 1\}<\varepsilon \quad \text { for all } x \in U \text {. }
$$

For $\varepsilon<1$, this forces $|f(x)-f(p)|<\varepsilon$ for all $x \in U$ and all $f \in F$. Thus $F$ is equicontinuous at $p$.

This theorem relates the equicontinuity of a given collection of functions $F$ on $X$ to the existence of a (uniformly bounded) family of continuous maps $\left\{a_{p} \mid p \in X\right\}$ on $X$. We denote this latter family by $A(F)$ and refer to it as the associated family of $F$. This relationship is critical in the next theorem and the definition that follows.

THEOREM 3. Let $X$ be a set and let $F \subset R^{X}$. Then there is a weakest topology on $X$ for which $F$ is an equicontinuous collection.

Proof. By Theorem 2, $F$ is equicontinuous if and only if the associated family is continuous. Hence the weak topology induced on $X$ by the collection $A(F)$ is necessarily the weakest topology on $X$ for which $F$ is equicontinuous.

Definition. The weakest topology on $X$ for which $F \subset R^{X}$ is equicontinuous is called the equiweak topology induced by $F$, and will be denoted by $\mathcal{T}^{F}$. By Theorem 3 it is, in fact, the weak topology induced on $X$ by the associated family $A(F)$, so that $\mathcal{T}^{F} \equiv \mathcal{T}_{A(F)}$.

EXAMPLE. The topology induced by a norm on a vector space is the equiweak topology induced by the unit ball of its continuous dual. Using our previous notation with $F$ taken to be the unit ball of the continuous dual, we have $a_{p}(x)=\sup _{F}\{|f(x)-f(p)| \wedge 1\}=\|x-p\| \wedge 1$, where \|\| denotes the given norm.

In the case of the supremum norm on $C(X)$, where $X$ is a compact space, we may take $F$ to be the family of evaluation maps on $C(X)$.

Before turning to the metrization problem, we record some elementary, but useful, observations about the equiweak topology and about the relationship between $F$ and $A(F)$.

Remark 1. For any $F \subset R^{X}, \mathcal{T}_{F} \subset \mathcal{T}^{F}$. Equality holds if and only if $F$ is equicontinuous in its weak topology.

In the proof of (i) $\Rightarrow$ (ii) in Theorem 2 , the neighborhood used to establish the 
continuity of $a_{p}$ at a point is independent of the choice of $p$, so the collection $A(F)$ is, in fact, equicontinuous. Thus we have

REMARK 2. $F$ is equicontinuous if and only if $A(F)$ is equicontinuous.

In particular, since $F$ is equicontinuous in $\mathcal{T}^{F}$ and $\mathcal{T}^{F} \equiv \mathcal{T}_{A(F)}$, we have

REMARK 3. $A(F)$ is equicontinuous in its weak topology.

Finally, it is straightforward to establish

ReMARK 4. $F$ separates points in $X$ if and only if $A(F)$ separates points in $X$.

Equiweak topologies and metrization. Recently, Guthrie and Henry [1], [2] have characterized pseudometrizable topologies in terms of weak topologies induced by subfamilies of $C(X)$ satisfying various conditions, one of which is $\sigma$-equicontinuity. A slight modification of the proof of their Theorem 4 of [2] yields the following characterization of pseudometrizability:

TheOREM 4. A topology for $X$ is pseudometrizable if and only if it is a weak topology induced by an equicontinuous family of real-valued functions.

Note that this characterization requires that the topology of $X$ be known a priori, for otherwise the notion of equicontinuity would be meaningless. By contrast, the concept of the equiweak topology allows us to identify, in terms of subfamilies of $R^{X}$ rather than $C(X)$, those topologies for $X$ which are pseudometrizable.

TheOREM 5. A topology for $X$ is pseudometrizable if and only if it is an equiweak topology induced by a family of real-valued functions.

Proof. By Theorem 4, a pseudometrizable topology $\mathcal{T}$ must be $\mathcal{T}_{F}$ for some equicontinuous $F$. But by Remark 1 , this implies $\mathcal{T}^{F}=\mathscr{T}_{F}=\mathcal{T}$, so $\mathcal{T}$ is an equiweak topology. Conversely, if $\mathscr{T}=\mathscr{T}^{F}$, then $\mathscr{T}=\mathscr{T}_{A(F)}$ where $A(F)$ is the associated family of $F$. But by Remark $3, A(F)$ is equicontinuous in $\mathcal{T}_{A(F)}=\mathcal{T}$, so Theorem 4 applies, and $\mathcal{T}$ is pseudometrizable.

In view of Theorem 5, we see that the equiweak topology provides a method of generating all pseudometric topologies on a set $X$ just as the weak topology provides a method of generating all completely regular topologies on a set $X$. Furthermore, just as the point-separating subfamilies of $R^{X}$ determine those completely regular topologies which are Hausdorff, those same subfamilies determine which pseudometrizable topologies are Hausdorff.

Theorem 6. A topology for $X$ is metrizable if and only if it is an equiweak topology induced by a point-separating family of real-valued functions.

Proof. If $d$ is a metric inducing the topology $\mathcal{T}$ of $X$, then the function $f_{x}$ : $X \rightarrow R$ defined by $f_{x}(y)=d(x, y)$ separates $x$ from each other point of $X$. Then $F=\left\{f_{x} \mid x \in X\right\}$ separates points and $\mathcal{T}^{F}=\mathcal{T}$. Conversely, if $F$ separates points in $X$, so does $A(F)$, by Remark 4, so the distance function defined by $\sup _{A(F)}\{|a(x)-a(y)|\}$ is, in fact, a metric which induces the topology $\mathcal{T}_{A(F)}=\mathscr{J}^{F}$.

In closing, we should point out to the reader, as the referee has to us, that the analogy between the role of weak topologies in the theory of completely regular spaces and the role of equiweak topologies in the theory of pseudometrizable 
spaces is imperfect. In particular, if $X$ is completely regular, then every subset of $C(X)$ will induce a weaker completely regular topology on $X$, whereas if $X$ is pseudometrizable, only the equicontinuous subsets of $C(X)$ will induce a weaker pseudometrizable topology on $X$ via the equiweak topology.

\section{REFERENCES}

1. J. A. Guthrie and M. Henry, Metrization, paracompactness, and real-valued functions, Fund. Math. 95 (1977), 49-54.

2. ___ Metrization, paracompactness, and real-valued functions. II, Fund. Math. 104 (1979), 13-20.

Department of Mathematics, West Virginia University, Morgantown, West Virginia 26506 